\title{
NASA Human Health and Performance Center: Open Innovation Successes and Collaborative Projects
}

\author{
Jeffrey R. Davis, MD $^{(1)}$, Elizabeth E. Richard ${ }^{(2)}$ \\ (1)NASA, 2101 NASA Parkway, Houston, Texas, 77058, 281.483.0393, jeffrey.r.davis@nasa.gov \\ (2)Wyle, 1290 Hercules Ave, Houston, Texas, 77058, 610.291.1862, erichard@wylehou.com
}

Keywords:

NHHPC, collaboration, innovation, health, engagement

\begin{abstract}
In May 2007, what was then the Space Life Sciences Directorate published the 2007 Space Life Sciences Strategy for Human Space Exploration, which resulted in the development and implementation of new business models and significant advances in external collaboration over the next five years. The strategy was updated on the basis of these accomplishments and reissued as the NASA Human Health and Performance Strategy in 2012, and continues to drive new approaches to innovation for the directorate. This short paper describes the open innovation successes and collaborative projects developed over this timeframe, including the efforts of the NASA Human Health and Performance Center (NHHPC), which was established to advance human health and performance innovations for spaceflight and societal benefit via collaboration in new markets.
\end{abstract}

\subsection{Introduction}

Severe budget cuts to the human research and development budget, coupled with a growing awareness that organizations outside of NASA were advancing human health and performance innovations at an increasingly rapid pace, drove the NASA Space Life Sciences Directorate to adopt a strategic plan in 2007 grounded on a new business model anchored in collaboration. Human space missions are now conducted for 6-month durations, and a one-year mission is planned for 2015. Given the diverse nature of the space flight population and increasing length of missions, ever more sophisticated medical care and environmental health systems and an infusion of novel approaches and technologies to improve health care in flight are required.

Since 2009, NASA has utilized collaboration and open innovation processes to seek solutions to some of the more difficult and pressing space medical care issues. In 2010, NASA established the NASA Human Health and Performance Center (NHHPC) as a forum to exchange best practices in innovative approaches, and to facilitate partnership development across sectors for solving medical, environmental, and human performance problems in space and on Earth. These novel approaches have resulted in significant advances to achieving NASA's human health and performance goals, as well as benefiting life on Earth.

\subsection{Discussion}

\subsubsection{Open Innovation}

To initiate open innovation efforts, NASA utilized a Harvard Business School process to determine which of its challenges in human health and performance research and technology development were most amenable to problem solving using the open innovation approach. The result was the identification of challenges for which problem statements were developed, including data-driven forecasting of solar events; food packaging to maintain quality for 5 years; compact (one cubic foot, 20 pound) exercise device for capsules; accurate tracking of medical consumables in flight.

Three open innovation platform providers were procured for the pilot study: 1) InnoCentive, which posts individual challenges/gaps to their established network of $~ 300,000$ solvers and offers a financial award if the solution is found viable by the posting entity; 2) yet2.com which acts as a technology scout bringing together buyers and sellers of technologies and results in the option to develop partnerships; and 3) TopCoder, an open innovation software company with a network of $\sim 300,000$ solvers and conducts a variety of skill-based software coding competitions. 
Results of the initial open innovation pilot projects with InnoCentive are presented in Table 1.0, including the number of proposed solutions submitted by the community, the amount of the award for the winning proposal (partial prizes may be awarded if requirements are partially met or if more than one viable solution is submitted), and the current status of the challenge. The InnoCentive solver community advanced the state of knowledge or provided solutions to problems as diverse as predicting a solar proton event to improve space flight operations, to long-duration food packaging, to compact exercise devices. In 2010 NASA procured InnoCentive as a platform through a competitive process, and has since used its solver community to evaluate ways for the non-invasive measurement of intracranial pressure, a problem that has emerged as a significant risk during long duration spaceflight. The results of this challenge are also shown on Table 1.0.

\begin{tabular}{|c|c|c|}
\hline \multicolumn{1}{|c|}{ Challenge } & Submissions & Award \\
\hline $\begin{array}{c}\text { Data-Driven Forecasting of Solar Events } \\
\text { Resulting model showed a high percent correct ( 95\%) but } \\
\text { with an equally high false alarm rate. Potential for } \\
\text { coupling with other modeling efforts. }\end{array}$ & 11 & $\$ 30,000$ \\
\hline $\begin{array}{l}\text { Food Packaging and Protection } \\
\text { Monitoring other packaging team evaluations of flexible } \\
\text { graphene material proposed as solution. }\end{array}$ & 22 & $\begin{array}{c} \\
(3 \text { partial) }\end{array}$ \\
\hline $\begin{array}{l}\text { Compact Aerobic Resistive Exercise Device Mechanism } \\
\text { Technology was included in Advanced Exercise Concepts } \\
\text { trade space for consideration. }\end{array}$ & 95 & $\$ 20,000$ \\
\hline $\begin{array}{l}\text { Non-invasive Measure of Intracranial Pressure } \\
\text { Resulted in a predictive algorithm from UCLA using } \\
\text { available physiologic data. Site visit planned to assess } \\
\text { UCLA analysis of NASA data via modification of existing } \\
\text { NSBRI study. }\end{array}$ & 638 & \\
\hline
\end{tabular}

Table 1.0, Select InnoCentive Results

The pilot competitions with yet2.com resulted in active leads for technical needs in three areas as shown on Table 2.0, introducing technological approaches not previously seen, providing enhanced insight regarding the marketplace, and informing strategies for investigating technologies that meet NASA's specialized needs. The TopCoder challenge resulted in an open source license to optimize a proposed lunar medical kit, which was incorporated into the current medical model. Since this time, NASA has increased its experience with open innovation resulting in solutions for algorithms that optimize an ECG signal on a smart phone so that field ECG recordings can be validated and sent to a medical provider.

\begin{tabular}{|c|c|c|}
\hline \multicolumn{1}{|c|}{ Technical Need } & Total Leads & Active Leads \\
\hline Hip Bone Microarchitecture Measurement & 51 & 5 \\
$>\quad \begin{array}{l}\text { Pilot study quantifying changes in sheep bone } \\
\text { microarchitecture for preclinical validation expected to be } \\
\text { completed by the end of FY13 }\end{array}$ & \\
$\begin{array}{l}\text { Provides foundation for a research proposal to validate this } \\
\text { technology in a population of spinal cord injured subjects }\end{array}$ & 61 & 8 \\
\hline $\begin{array}{l}\text { Water Disinfection and Monitoring } \\
\quad \begin{array}{l}\text { Provided a status of state-of-the-art water disinfection and } \\
\text { monitoring alternatives, which indicated a need for NASA } \\
\text { to develop new technologies for our specialized needs } \\
\text { during spaceflight }\end{array}\end{array}$ & \\
\hline Food Packaging and Protection & 29 & 5 \\
\hline
\end{tabular}




\begin{tabular}{|l|l|l|}
\hline$>$ Evaluation of one lead as partner underway & & \\
\hline
\end{tabular}

Table 2.0, Select yet2.com Results

\subsubsection{NASA Human Health and Performance Center (NHHPC)}

\subsubsection{Engagement}

The 2007 Space Life Sciences Strategy stated an objective to establish a virtual center to advance human health and performance innovations benefiting life in space and on Earth, and in 2010 the NASA Human Health and Performance Center (NHHPC) was launched to meet this objective. The center promotes new ways of doing business, shares best practices among member organizations, and engages in collaborative projects to leverage resources. The NHHPC, which now boasts over 135 corporate, government, academic and non-profit members, has convened four successful workshops and engaged in multiple collaborative projects, and will conduct its fifth workshop in Houston in June 2014. The center is also developing a streamlined partner engagement process to capture technical needs and opportunities of NHHPC members, facilitate partnership development, and establish and manage collaborative projects for NASA.

The NHHPC provides a forum for member organizations worldwide to interact on multiple topics such as mobile health, the theme of the 2012 NHHPC workshop. The "mHealth - Smart Media and Health: Applications Benefiting Life in Space and on Earth" workshop for NHHPC members addressed mobile health applications benefitting global and space health issues, including technologies for consumer driven healthcare mobile access, democratizing health diagnostics, and a variety of smartphone applications. The most recent NHHPC workshop was conducted in November 2013 on the topic of "Accelerating Innovation: New Organizational Business Models," and focused on cross-sector and other collaborative approaches successfully used by organizations to achieve their goals. The 2014 workshop will again bring together NHHPC member organizations from around the world to address the topic of "Innovation through Co-development: Engaging Partners" in conjunction with the Houston Technology Center and the National Space Biomedical Research Institute (NSBRI).

To further engage NHHPC members and facilitate partnership development for NASA, the NHHPC is creating a partner engagement process that will be deployed in 2014. This comprehensive process addresses opportunities from multiple points of intake through a common format on the NHHPC website and leverages the existing NASA exploration medical capability technology watch process by automatically routing project opportunities for evaluation to assigned subject matter experts. The new process will also incorporate the Solution Mechanism Guide, a project and decision management tool under development by the NASA Human Health and Performance Directorate. The result is an end-to-end electronic process that spans identification of a potential opportunity, to selecting the most effective tool to implement a project, to providing project status updates. Two projects have been enabled by this evolving process to date.

\subsubsection{Collaborative Projects}

The NHHPC facilitates collaborative project development among its member organizations focusing on applications that have societal benefits as well as addressing needs for human spaceflight. One project underway among NHHPC members is the pursuit of one to several novel strategies to increase medication stability that would enable health care in remote terrestrial settings as well as during space flight. This project is being coordinated by NASA and the Health and Environmental Sciences Institute (HESI), and will include pharmaceutical companies and other nonprofit organizations. Wherever possible, the NHHPC seeks to find common ground among many different sectors and members to drive innovation.

NASA has signed Space Act Agreements with multiple corporate NHHPC partners including GE, Philips, and Nike, and is pursuing projects that meet the missions of NASA and the partner organizations. The GE NHHPC collaboration is seeking to develop ultrasound methodologies to examine the eye that will enable NASA to further understand the eye changes related to long-duration space flight. The adaptation of ultrasound to this type of eye examination could also expand the use of ultrasound in health care on the Earth in settings where MRIs are not available. 
The Nike collaboration is aimed at exploring possible adaptation of the Nike+ Hyperworkout shoe for use during exercise sessions on the International Space Station (ISS). Crewmembers aboard ISS are required to perform a wide variety of resistive exercises to protect the musculoskeletal system against microgravity-induced atrophy using the Advanced Resistive Exercise Device (ARED). The ARED instrumentation box and force plates, meant to display exercise prescriptions and record the exercise loads and repetitions performed by each crewmember, have been out of service since 2011 and will not be repaired for some time. As a result, the crew must manually complete exercise logs that are monitored for adherence to exercise prescriptions and strength adaptations, and to advance design of exercise programs. The Nike+ shoe is being evaluated as a surrogate force monitoring system while the ARED is being repaired. Under the partnership, NASA and Nike will examine the possible use of data from the instrumented workout shoes as a means to monitor ARED exercise sessions.

NASA has further developed several mobile health projects through the NHHPC. One mobile health application developed by Netra can perform eye refraction exams that could be utilized in space flight as well as in remote settings on Earth. Another is the development of a dry-electrode vest that can send an ECG signal wirelessly to a smartphone. For this project, NASA also used open innovation platforms to run an algorithm contest to optimize the ECG algorithm on a smartphone, so that a non-medical user could be assured the ECG signal was valid.

NHHPC projects may also focus on collaborative research. NASA and the National Science Foundation (NSF) are currently proposing a joint study on the biology of stress using an accelerated research model called Ideas Lab. Ideas Lab is a facilitated approach that combines multiple research disciplines around a single topic and generates creative research proposals in a rapidly iterative process. NASA and the NSF conducted a workshop in December 2013 to develop topics of mutual interest regarding the biology of stress. This joint Ideas Lab could then combine the research talents of both organizations leading to findings of benefit to space flight and health care on Earth.

\subsection{Conclusion}

Through this short paper, the authors have described the NASA Human Health and Performance Directorate's new business model to advance research and technology innovations. Beginning in 2007, novel approaches based on collaboration have been implemented to not only advance the NASA mission, but also to address global problems in human health. The use of open innovation has resulted in significant advances in achieving NASA's human health and performance goals, and the development of virtual partnership centers, such as the NHHPC, has greatly facilitated project development among partners and the rapid sharing of best practices and results for the benefit of all. 